\title{
Cholecystectomy as a risk factor of metabolic syndrome: from epidemiologic clues to biochemical mechanisms
}

\author{
Yongsheng Chen, Shuodong Wu and Yu Tian
}

Cholecystectomy has long been regarded as a safe procedure with no deleterious influence on the body. However, recent studies provide clues that link cholecystectomy to a high risk for metabolic syndrome (MetS). In the present review, we describe the epidemiologic evidence that links cholecystectomy to MetS. Various components of MetS are investigated, including visceral obesity, dyslipidemia, elevated blood pressure, impaired fasting glucose, and insulin resistance. The possible mechanisms that associate cholecystectomy with MetS are discussed on the basis of experimental studies. Laboratory Investigation (2018) 98, 7-14; doi:10.1038/labinvest.2017.95; published online 11 September 2017

Gallstone disease is one of the most common biliary tract diseases in which both environmental and genetic factors have roles in its pathogenesis. Biliary cholesterol supersaturation from metabolic defects in the liver and the subsequent physical-chemical imbalance of cholesterol solubility in bile are thought to be the main cause of gallstone formation. ${ }^{1}$ Factors such as physical inactivity, obesity, and overnutrition increase the risk of developing gallstones and form the basis of primary prevention by lifestyle changes. ${ }^{2}$ Although treatment of asymptomatic gallstone patients is not routinely recommended, current treatment algorithms for symptomatic gallstone patients, by contrast, remain predominantly invasive and are based on surgery. ${ }^{3}$

Cholecystectomy is the best and most cost-effective treatment for gallstone disease, and one of the most commonly performed operations worldwide. The short- and long-term outcomes of cholecystectomy are excellent, and the surgical procedure has minimal rates of mortality and morbidity. It is also accepted that gallbladder (GB) removal is a relatively innocuous procedure with no deleterious influence on bile acid (BA) metabolism or overall metabolic regulation. Following surgery, the size of the BA pool remains within a normal range and dietary fat absorption remains unaffected. ${ }^{4,5}$

However, there is emerging evidence showing that cholecystectomy itself may lead to an excessive risk for the surgical patient developing metabolic disorders and their associated complications, including dyslipidemia, nonalcoholic fatty liver disease (NAFLD), and hyperglycemia. ${ }^{5-7}$ The unexplained high incidence of these metabolic abnormalities among cholecystectomized patients, combined with the lack of data regarding metabolic changes that occur after GB removal, has caused cholecystectomies to receive increased scrutiny. As the roles of the GB and BAs in systemic metabolic regulation have become better understood, ${ }^{8-10}$ it is important to re-examine the potential long-term negative outcomes of GB removal, and especially the associated risk for subsequent metabolic syndrome (MetS). In the present review, we describe the epidemiologic evidence that links cholecystectomy to MetS, and discuss some possible mechanisms for this linkage based on results of experimental studies.

\section{METABOLIC SYNDROME}

MetS refers to a constellation of metabolic disorders that include central obesity, dyslipidemia (raised triglycerides (TGs) and lowered high-density lipoprotein cholesterol), raised blood pressure, and raised fasting glucose, and can be diagnosed when any three of the above five components are present $^{11}$ (Table 1). The prevalence of MetS has increased over time, and data gathered by the National Health and Nutrition Examination Survey (NHANES) indicate that $\sim 20 \%$ of the adult US population is currently affected by MetS. ${ }^{12}$ In addition, there is a high prevalence of MetS and its individual components in developing countries. ${ }^{13,14}$ It is notable that MetS adversely affects the health of increasing numbers of adolescents, as $\sim 50 \%$ of them display at least one of its components. ${ }^{15}$ Because MetS directly promotes the development of cardiovascular diseases, type 2 diabetes 
Table 1 Criteria for diagnosis of metabolic syndrome

\begin{tabular}{ll}
\hline Measure & Categorical cutpoints \\
\hline Waist circumference & Population- and country-specific definitions \\
Triglycerides & Triglycerides $\geq 150 \mathrm{mg} / \mathrm{dl}$ or drug treatment for elevated triglycerides \\
High-density lipoprotein cholesterol (HDL-C) & $\mathrm{HDL}-\mathrm{C}<40 \mathrm{mg} / \mathrm{dl}$ in men or $<50 \mathrm{mg} / \mathrm{dl}$ in women or drug treatment for reduced HDL-C \\
Blood pressure & Systolic BP $\geq 130 \mathrm{~mm} \mathrm{Hg}$, diastolic BP $\geq 85 \mathrm{~mm} \mathrm{Hg}$ or antihypertensive drug treatment in a patient with history \\
& of hypertension \\
Fasting glucose & $\geq 100 \mathrm{mg} / \mathrm{dl}$ or drug treatment for elevated glucose \\
\hline
\end{tabular}

Source: 2009 Joint Interim Statement of the International Diabetes Federation Task Force on Epidemiology and Prevention; National Heart, Lung, and Blood Institute; American Heart Association; World Heart Federation; International Atherosclerosis Society; and International Association for the Study of Obesity. ${ }^{11}$

mellitus (T2DM), stroke, and chronic kidney disease, ${ }^{16-18}$ it has become a major public-health challenge worldwide. ${ }^{19,20}$

Despite years of investigation, the cause of MetS remains obscure. However, several risk factors are probably involved, with visceral obesity and insulin resistance being the most important. ${ }^{21,22}$ An association between gallstone disease and MetS was first reported by Mendez-Sanchez et al in 2005, ${ }^{23}$ and their findings were confirmed by two other large sample studies. ${ }^{24,25}$ Moreover, patients with complicated gallstone diseases, which often require cholecystectomy, are more likely to have MetS than those with uncomplicated gallstone disease. ${ }^{26}$ In a cross-sectional study, Shen et al. analyzed data from 5672 subjects, and found that the prevalence of MetS was $53.2 \%$ among the subjects with gallstone disease and $63.5 \%$ among cholecystectomized subjects. ${ }^{27}$ These findings suggest that cholecystectomy by itself may be a risk factor for MetS.

\section{CHOLECYSTECTOMY AND MetS COMPONENTS}

Numerous epidemiological studies have linked gallstones and cholecystectomy to MetS. However, when compared with gallstones, cholecystectomy is usually more closely, if not solely, associated with each of the MetS components when the two conditions are studied separately.

An earlier study ${ }^{28}$ reported a significant weight gain among cholecystectomized patients, with men and women gaining a mean of $4.6 \%$ and $3.3 \%$, respectively, of their preoperative body weight at 6 months after surgery. Other studies ${ }^{27,29}$ that compared subjects with and without gallstones reported that, when compared with subjects without gallstones, the body mass index (BMI) and waist circumference of subjects with gallstones tended to be higher, and these differences were even more pronounced in cholecystectomized subjects (Table 2). The tendency for an increase in BMI after cholecystectomy was not seen in patients who had undergone nonbiliary surgery. ${ }^{30}$ This weight gain was generally presumed to be caused by a return to the high-fat dietary habits that contributed to development of their cholelithiasis. ${ }^{31}$ However, metabolic causes may have been a contributing factor, as there was no significant difference in weight gain between patients who remained on their preoperative low-fat diet and those who did not, with the former group showing an increase of $2 \mathrm{~kg} / \mathrm{m}^{2}$ in their mean BMI. ${ }^{30}$ Obesity increases the likelihood of developing various diseases, including hypertension, coronary heart disease, fatty liver disease, and T2DM. Further increases in BMI can adversely affect the long term health of these patients, because many of them are overweight prior to surgery.

In an attempt to investigate the effect of cholecystectomy on hepatic lipid metabolism, Amigo et al. ${ }^{5}$ reported significant increases in serum and hepatic TG concentrations, hepatic very-low-density lipoprotein (VLDL)-TG levels, and apoB-48 production in mice 2 months after cholecystectomy surgery. Interestingly, those mice also displayed an altered rhythm of changes in their hepatic TG concentrations when compared with sham-operated animals, by having higher TG concentrations at the beginning of the light phase of each diurnal cycle, and then maintaining those high concentrations throughout the day. Consistent with findings in mice, significant increases in VDLD-apoprotein B (VLDL-apoB) and intermediate-density lipoprotein apoprotein B concentrations were noticed in patients 3 years after cholecystectomy. ${ }^{32}$ In another case-control study, patients who underwent cholecystectomy had higher levels of plasma TGs, as well as higher levels of total and low-density lipoprotein cholesterol, but lower levels of high-density lipoprotein cholesterol than control subjects without GB disease or who had not undergone cholecystectomy $^{33}$ (Table 2). These observations support a still unknown role for the GB in regulating systemic lipid metabolism, and suggest that GB removal may somehow result in a deranged lipid profile and stimulate accumulation of fat in the liver. Thus, it is reasonable to speculate that cholecystectomized patients are more likely to develop fatty liver disease when exposed to the metabolic stress produced by a high-fat diet.

As expected, Nervi et $a^{29}$ found a higher prevalence of fatty liver among cholecystectomized subjects than among those with gallstones in a high-risk Hispanic population $(33.7 \%$ vs 
Table 2 Epidemiologic evidence that links cholecystectomy to various components of MetS

\begin{tabular}{|c|c|c|c|}
\hline $\begin{array}{l}\text { First author/ } \\
\text { (year)/[ref.] }\end{array}$ & $\begin{array}{l}\text { Population } \\
\text { studies }(n)\end{array}$ & Major findings & Comment \\
\hline Houghton et $a^{28}$ & British (103) & $\begin{array}{l}\text { Weight gain } 6 \text { months after cholecystectomy (4.6\% for men and } \\
3.3 \% \text { for women) }\end{array}$ & - \\
\hline Shen et $a 1^{27}$ & Chinese (5672) & $\begin{array}{l}\text { (1) BMl and waist circumference increase after cholecystectomy } \\
\text { ( } 26.02 \text { vs } 24.53 \mathrm{~kg} / \mathrm{m}^{2} \text { and } 90.5 \text { vs } 86.1 \mathrm{~cm} \text { ). } \\
\text { (2) Cholecystectomized subjects had higher levels of fasting } \\
\text { blood glucose than subjects with gallstones } \\
\text { (5.10 vs } 4.92 \mathrm{mmol} / \mathrm{l}, P<0.001)\end{array}$ & $\begin{array}{l}\text { Cholecystectomy significantly increases the risk } \\
\text { of metabolic syndrome }\end{array}$ \\
\hline Amigo et $a l^{5}$ & Mice & $\begin{array}{l}\text { TG, VLDL-TG, and apoB-48 increase after cholecystectomy } \\
(25 \%, P<0.02 ; 15 \%, P<0.03 ; 50 \%, P<0.01)\end{array}$ & GB has a role in hepatic TG regulation \\
\hline Juvonen et $a^{\beta 2}$ & Finnish (35) & $\begin{array}{l}\text { VLDL-apoB and IDL-apoB increase after cholecystectomy } \\
(P=0.0019 \text { and } P=0.0001 \text {, respectively })\end{array}$ & $\begin{array}{l}\text { Altered enterohepatic metabolism of cholesterol } \\
\text { after gallbladder removal }\end{array}$ \\
\hline $\begin{array}{l}\text { Chavez-Tapia } \\
\text { et al }\end{array}$ & Mexican (798) & $\begin{array}{l}\text { (1) Cholecystectomized patients had higher levels of plasma } \\
\text { TGs, total cholesterol, and LDL-cholesterol, but lower levels } \\
\text { of HDL-cholesterol than controls ( } 166 \pm 114 \text { vs } 152 \pm 90 \mathrm{mg} / \mathrm{d} \text {, } \\
P=0.04 ; 203 \pm 41 \text { vs } 181 \pm 47 \mathrm{mg} / \mathrm{d}, P<0.0001 ; 127 \pm 34 \text { vs } \\
101 \pm 41 \mathrm{mg} / \mathrm{d}, P<0.0001 ; 47 \pm 14 \text { vs } 55 \pm 37 \mathrm{mg} / \mathrm{d}, P<0.0001 \text { ). } \\
\text { (2) A higher prevalence of T2DM was found among } \\
\text { cholecystectomized patients than controls ( } 7.2 \% \text { vs } 3.2 \%, P=0.01 \text { ) }\end{array}$ & $\begin{array}{l}\text { Cholecystectomy increased the prevalence of } \\
\text { metabolic risk factors for cardiovascular disease }\end{array}$ \\
\hline Nervi et al ${ }^{29}$ & Chilean (881) & $\begin{array}{l}\text { (1) Prevalence of fatty liver is higher in cholecystectomized } \\
\text { subjects than in those with gallstones ( } 33.7 \% \text { vs } 27.0 \%) \text {. } \\
\text { (2) Cholecystectomy was more strongly related to T2DM than } \\
\text { was gallstones ( } 15.6 \% \text { vs } 11.2 \%) \text {. } \\
\text { (3) A significantly higher prevalence of hypertension in } \\
\text { cholecystectomized patients ( } 36.8 \% \text { vs } 18.7 \%, P<0.01 \text { ) }\end{array}$ & $\begin{array}{l}\text { Cholecystectomy is more closely associated with } \\
\text { MetS components }\end{array}$ \\
\hline Ruhl et al6 & American (12 232) & $\begin{array}{l}\text { NAFLD was associated with cholecystectomy, but not } \\
\text { gallstones (OR: } 2.4 ; 95 \% \mathrm{Cl}: 1.8-3.3)\end{array}$ & $\begin{array}{l}\text { Cholecystectomy may itself be a risk factor for } \\
\text { NAFLD }\end{array}$ \\
\hline Kwak et aß6 & $\begin{array}{l}\text { South Korean (17 } \\
\text { 612) }\end{array}$ & $\begin{array}{l}\text { Cholecystectomy, but not gallstones, is independently } \\
\text { associated with NAFLD } \\
\text { (cholecystectomy: OR: } 1.35,95 \% \mathrm{Cl}: 1.03-1.77, P=0.028 \text {; } \\
\text { gallstones: OR: } 1.15,95 \% \mathrm{Cl}: 0.95-1.39, P=0.153 \text { ) }\end{array}$ & \\
\hline Sonne et al & Danes (20) & $\begin{array}{l}\text { Elevated postprandial plasma glucose excursions in patients } \\
3-6 \text { months after cholecystectomy }(1431 \pm 31 \mathrm{vs} \\
1313 \pm 36 \mathrm{mmol} / \mathrm{l} / \mathrm{min}, P=0.023)\end{array}$ & $\begin{array}{l}\text { Deterioration of postprandial glycemic control } \\
\text { after GB removal, probably because of metabolic } \\
\text { changes }\end{array}$ \\
\hline
\end{tabular}

27.0\%). Their conclusion supports the results of another study that showed a significant increase in the development of hepatic steatosis at 3 months after cholecystectomy, regardless of whether heavy drinking occurred. ${ }^{34}$ In accordance with the above findings, a US cohort study based on the NHANES III showed that NAFLD, a hepatic manifestation of MetS, was associated with cholecystectomy, but not gallstones, even after controlling for multiple factors common to both disorders. ${ }^{6}$ This association was stronger in men than in women, as $68 \%$ of the cholecystectomized men had NAFLD. Moreover, cholecystectomized individuals were more likely to have elevated serum concentrations of alanine aminotransferase or $\gamma$-glutamyl transferase than individuals who had not undergone cholecystectomy (Table 2). In contrast, no independent association was found between the serum levels of liver enzymes and cholelithiasis. ${ }^{27,33,35}$ The association between cholecystectomy and elevated liver enzymes was much stronger if the cholecystectomy occurred $>10$ years, but was nonsignificant in patients within 10 years of the surgery. Furthermore, subjects with a history of cholecystectomy were 
twice as likely to be hospitalized for or die of cirrhosis as those who had not undergone cholecystectomy. The time interval between cholecystectomy and development of abnormal liver function is best explained by a causal relationship between the two factors. Ablation of the GB may favor the development of chronic disease conditions, including NAFLD, abnormal liver function, and cirrhosis. Cholecystectomy, rather than cholelithiasis, is a risk factor for the above-mentioned abnormalities. $^{36}$

Gallstone disease and T2DM share some important risk factors. Although there is evidence suggesting an increased risk of T2DM among patients with gallstone disease, the prevalence of gluco-metabolic disturbances in cholecystectomized patients is also unexpectedly high. Unfortunately, few studies have compared the importance of cholecystectomy and gallstones with regards to impaired glucose tolerance/ T2DM. Recently, Shen et al ${ }^{27}$ showed that cholecystectomized subjects had higher levels of fasting blood glucose than subjects with gallstones. Their observation was confirmed by Sonne et $a l,{ }^{7}$ who reported elevated postprandial plasma glucose (PG) excursions in patients who had undergone cholecystectomy within the previous 3-6 months. Those patients also had higher mean postprandial PG concentrations at $60 \mathrm{~min}$ after ingesting a mixed meal, and the levels remained elevated throughout the next $2 \mathrm{~h}$. The cholecystectomized patients also exhibited marginally elevated basal concentrations of glucagon and slightly lower postprandial concentrations of insulin. Those findings suggest the presence of an early prediabetic state in patients after GB removal. In agreement with the above results, a higher prevalence of T2DM was found among cholecystectomized patients than among subjects without gallstones or a history of cholecystectomy. ${ }^{33}$ Not surprisingly, when examined separately, cholecystectomy was proven to be more strongly related to T2DM than was gallstones ${ }^{29}$ (Table 2).

Consistent with the above findings, several lines of evidence suggest that cholecystectomy per se may increase the risk for developing hypertension. In a Chinese population of 5672 subjects, the prevalence of elevated blood pressure was higher among subjects with a history of cholecystectomy than among those with gallstones or without gallstone disease. ${ }^{27}$ Similarly, Chavez-Tapia et $a l^{33}$ analyzed the association between cholecystectomy and the risk factors for cardiovascular disease, and found that patients who underwent cholecystectomy had higher systolic blood pressure levels than those without GB disease or a history of cholecystectomy. Furthermore, Nervi et $a^{29}$ found a significantly higher prevalence of hypertension among subjects having undergone cholecystectomy when compared with patients without gallstone disease.

Collectively, the above data strongly suggest that cholecystectomy by itself is a risk factor for MetS, which is in line with the recently disclosed role of the GB in regulating metabolism. Thus, rather than being an innocuous procedure, cholecystectomy may have a major impact on an individual's overall metabolic regulation and health status. The propensity for cholecystectomized patients to be affected by metabolic disorders suggests that this risk should be discussed during preoperative counselling. In addition, long-term postoperative follow-up may be necessary to facilitate early health-care interventions, as cholecystectomized patients may develop comorbidities known to be associated with MetS.

The above-mentioned studies reveal a previously unrecognized fact that patients with a history of cholecystectomy differ from those with gallstones in the aspect of their systemic metabolism. The pathogenesis of gallstone disease was previously thought to result from defective liver metabolism, which remains unchanged after GB removal. However, recent studies have increased our knowledge of GB function and its essential role in the development of cholesterol gallstone disease. ${ }^{37}$ Thus, when conducting future epidemiological studies, it may be preferable to analyze cholecystectomized and gallstone patients separately with regards to their risk for developing MetS.

\section{MECHANISMS}

The underlying mechanisms by which cholecystectomy might cause MetS are not fully elucidated. However, an overview of our current understanding of GB physiology may provide some important insights.

The major function of the GB is to concentrate and store bile, mainly by absorbing water and ions via its epithelial cells. In this manner, hepatic bile is concentrated four- to fivefold by the normal-fasting GB. This leads to partitioning of a large portion of the BA pool within the GB, and increases the 'digestive power' of bile. The GB has motor function that allows for $20-30 \%$ emptying at 1 - to 2 -h intervals during a fasting state and $70-80 \%$ emptying after a meal. The interplay between $\mathrm{GB}$ contraction and relaxation has an important role in modulating the flow of BAs in enterohepatic circulation, which is responsible for regulating the whole-body homeostasis of TGs, BAs, and cholesterol. ${ }^{38,39}$

After cholecystectomy, the reservoir formed by the GB is suppressed, and hepatic bile is continuously secreted into the duodenal lumen. As a result, the BA pool circulates more quickly, exposing enterohepatic organs and eventually peripheral tissues to a higher flux of BAs during fasting periods of the diurnal cycle. ${ }^{5}$ This may exert a profound influence on the entire organism, given the novel findings that BAs may act as hormonal signals via their interactions with several enterohepatic and peripheral target receptors. $8,9,40-42$ Among these receptors, the farnesoid $\mathrm{X}$ receptor (FXR), a major BA-responsive ligand-activated transcription factor involved in regulating energy expenditure and glucose homeostasis, ${ }^{43-46}$ and the G-protein-coupled BA receptor (GPBAR1/TGR5), a cell-surface receptor ubiquitously expressed to various degrees in numerous human and rodent tissues, ${ }^{47-49}$ are the best studied. FXR is involved in the regulation of energy expenditure, lipid metabolism, and glucose homeostasis. For example, FXR activation can 


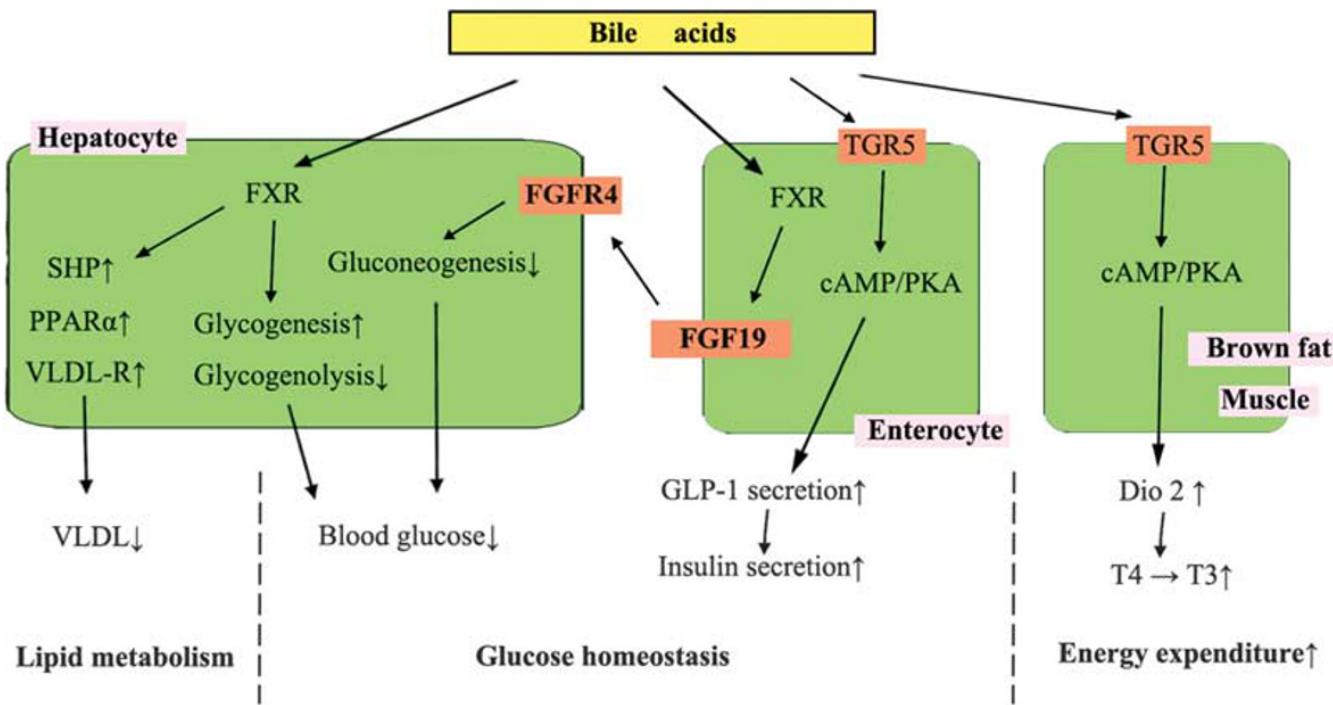

Figure 1 Function of BAs in metabolic regulation: FXR-mediated and GPBAR1/TGR5-mediated signaling pathways. In hepatocytes, activation of FXR by BAs can upregulate SHP, PPARa, and VLDL-R, which reduces lipogenesis and enhances lipolysis. As a result, plasma VLDL level is decreased. FXR also increases glycogenesis and decreases glycogenolysis. In enterocytes, FXR signaling upregulates FGF19 expression. FGF19 then circulates to the liver and inhibits gluconeogenesis through binding and activation of FGFR4. The activation of TGR5 by BAs stimulates GLP-1 secretion from enteroendocrine $L$ cells, which increases insulin secretion from pancreatic $\beta$ cells and improves insulin resistance. In brown fat and skeletal muscle cells, TGR5 activation leads to increased intracellular CAMP levels and Dio 2 expression. Dio 2 converts inactive thyroxine (T4) to active 3,5,3'-triiodothyronine (T3) to increase energy expenditure. BA, bile acid ; Dio 2, iodothyronine deiodinase type 2; FGFR4, FGF receptor 4; FXR, farnesoid X receptor GLP-1, glucagon-like peptide-1; PPARa, peroxisome proliferator-activated receptor $a$; SHP, small heterodimer partner; VLDL-R, very-low-density lipoprotein-receptor.

improve insulin sensitivity and reduce PG level by decreasing gluconeogenesis and increasing glycogen synthesis. Concordantly, FXR-null mice display elevated serum glucose levels and impaired insulin tolerance. GPBAR1, also known as TGR5, is activated by both primary and secondary BAs. Several lines of evidence suggest that this receptor has important metabolic effects. BAs could signal through the cell surface GPBAR1/TGR5 in brown fat and skeletal muscle cells to increase energy expenditure and prevent diet-induced obesity. ${ }^{10}$ In agreement with this observation, targeted disruption of the Gpbarl/TGR5 gene in mice favors fat accumulation with body weight gain, which is proved to be the result of decreased energy expenditure. ${ }^{50}$ A link between circulating levels of BAs and glucose metabolism had been shown by multiple studies, which demonstrated that BAs regulate glucose homeostasis through activation of GPBAR1/TGR5. ${ }^{51-53}$ In the small intestine, GPBAR1/TGR5 activation could favorably modulate insulin sensitivity and glucose metabolism through stimulating the secretion of the incretin hormone glucagon-like peptide-1 (GLP-1) by ileal enteroendocrine L cells. ${ }^{54,55}$ In line with above observation, $\mathrm{TGR}^{-1-}$ mice showed a delayed glucose clearance compared with their wild-type littermates. Taken together, the activation of FXR and GPBAR1/TGR5 by BAs is thought to favorably regulate lipid metabolism, glucose homeostasis, and energy expenditure via complex and inter-related pathways, whose details are beyond the scope of this review (Figure 1).
As an extension of these ideas, enhanced activation of the above receptors as a result of increased BA concentrations should theoretically benefit human metabolism. However, Amigo et al found that this was not true, as an essential factor was overlooked in the previous studies. The remarkable effect of GB removal is reflected in changes in the rhythm and intensity of BA flow rather than in the mean hepatic BA output (flux of BA molecules to the intestine per unit time) and the mean BA pool circulation rate. As a large portion of the BA pool is partitioned in the GB during a fasting state, a healthy functional GB is capable of increasing the intraduodenal BA concentration by a factor of 10 after a meal. In contrast, cholecystectomized subjects are not capable of eliciting a rapid release of BAs into the duodenum in response to food intake. As a result, their intraduodenal BA flow exhibits a flat curve rather than the wavy curve displayed by subjects with an intact GB. ${ }^{7}$ Pathological changes in the synchrony between BA circulation kinetics and periodic food intake, resulting from GB removal, may alter the rhythm of receptor activation, resulting in metabolic abnormalities. As expected, both glucose and lipid responses in cholecystectomized subjects become exaggerated after a meal. If BAs act as hormonal signals that exert a beneficial influence on metabolism, cholecystectomized subjects would be unable to promptly elicit a protective response to balance the sharp fluctuations in glucose and lipid levels induced by food intake; this inability might result in certain pathophysiological conditions. According to this view, the function of the GB 


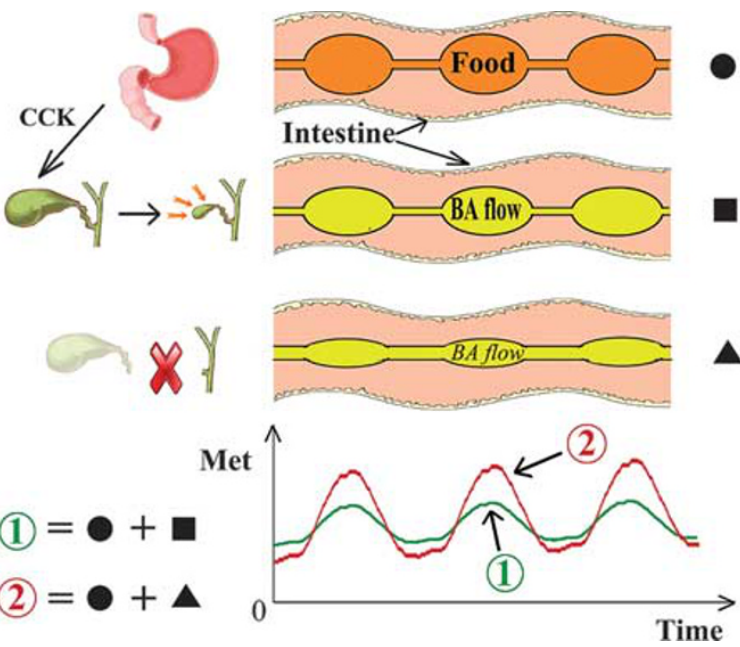

Figure 2 Possible mechanisms linking GB motor function to metabolism and cholecystectomy to metabolic syndrome. •: periodical food intake. BA flow under normal GB function. The rhythm and intensity of BA flow are in synchrony with periodical food intake. $\mathbf{A}$ : BA flow after GB removal. Although the basal BA output is higher than normal, the release of BAs is not enough to balance the metabolic stress caused by food intake. (1): Fluctuation in blood glucose or lipid concentrations under normal state. (2): Fluctuation in blood glucose or lipid concentrations after GB removal. BA, bile acid; GB, gallbladder; Met, blood glucose or lipid concentrations.

is not only to provide 'greater digestive power,' but also to allow GB bile to better balance the metabolic stress caused by periodic food intake (Figure 2). This point of view is also supported by phylogeny, as the presence of a GB appears to be a primitive trait. As reviewed by Oldham-Ott et $a,^{56}$ the presence or absence of a GB is mainly dependent on an animal's feeding rhythm. Animals that eat almost continuously, such as pigeons, deer, and rats, do not possess a GB, whereas carnivores (except whales), which eat only periodically, invariably have a GB.

Alternatively, events occurring within the GB itself may provide another possible mechanism for the increased risk of metabolic derangements following cholecystectomy. The GB has a metabolic function. As recently discovered, the GB mucosa is the main enterohepatobiliary site for expression of fibroblast growth factor 19 (FGF19), which is secreted at high concentrations into bile. ${ }^{57}$ Unlike its counterparts, FGF19 can function as an endocrine hormone and exert metabolic effects in tissues at distant locations. In this manner, FGF19 assists in regulating lipid, glucose, and energy metabolism during fed and fasted states. ${ }^{58-60}$ Mice treated with FGF19 exhibit reduced levels of serum TGs and cholesterol, as well as increased glycogen synthesis. FGF19 is capable of suppressing insulin-induced fatty-acid synthesis in the liver, which is a key process that controls glucose tolerance and triacylglycerol accumulation in liver, blood, and adipose tissue. ${ }^{61}$ A previous study $^{58}$ showed that transgenic mice expressing human FGF19 did not become obese or diabetic when challenged with a high-fat diet because they had increased levels of energy expenditure and insulin sensitivity. Accordingly, pharmacological administration of FGF19 to mice increases their metabolic rate and attenuates diet-induced obesity; furthermore, it also reverses diabetes in leptin-deficient mice. ${ }^{59}$ Thus, it is plausible to postulate that ablation of the GB could produce a marked decrease in the circulating levels of FGF19, and render such patients more prone to developing metabolic disorders. Interestingly, there is evidence that serum FGF19 concentrations decrease in humans after GB removal. ${ }^{62}$ Moreover, both the epithelial and smooth muscle layers of the $\mathrm{GB}$ express the membrane-bound $\mathrm{BA}$ receptor Gpbar1/TGR5 at a much higher level than any other tissue in the body. ${ }^{63}$ Thus, removal of the GB should theoretically result in a marked suppression of Gpbar1/TGR5-dependent metabolic signaling emanating from the GB, and a subsequent systemic influence on metabolic homeostasis.

\section{SUMMARY}

Cholecystectomy, a surgery that usually produces instant relief for patients with symptomatic cholelithiasis, has long been regarded as a safe procedure by both the general population and health-care professionals. Moreover, adoption of the laparoscopic approach for cholecystectomy has made it one of the most successful major abdominal procedures worldwide. However, increasing evidence contradicts this common wisdom, by showing that cholecystectomy is associated with an increased risk for long-term postoperative adverse events, such as an increased risk for developing cancer. In this review, additional evidence has been provided, suggesting that cholecystectomy may dysregulate the homeostasis of whole-body metabolism. If this is true, cholecystectomy may not be the innocuous procedure previously believed, but rather may adversely affect the metabolic balance of the entire body. The increasing prevalence of MetS, particularly among cholecystectomized patients, highlights the urgency for addressing the actual cause-effect relationship in future prospective epidemiological and interventional studies. Attempts to investigate the GB's metabolic function may improve our understanding of the mechanisms behind the connection between GB removal and MetS, and how the endocrine system functions in cholecystectomized patients.

\section{ACKNOWLEDGMENTS}

This work was supported by the NSFC (Natural Science Foundation of China; 81500483) and also by Liaoning Province Science and Technology Project (2013225049). The authors would like to thank Megan Khu for critical discussions in written-language.

\section{DISCLOSURE/CONFLICT OF INTEREST}

The authors declare no conflict of interest.

1. Portincasa P, Moschetta A, Palasciano G. Cholesterol gallstone disease. Lancet 2006;368:230-239.

2. Lammert F, Gurusamy K, Ko CW, et al. Gallstones. Nat Rev Dis Primers 2016;2:16024.

3. Portincasa P, Di Ciaula A, Wang HH, et al. Medicinal treatments of cholesterol gallstones: old, current and new perspectives. Curr Med Chem 2009;16:1531-1542. 
4. Kullak-Ublick GA, Paumgartner G, Berr F. Long-term effects of cholecystectomy on bile acid metabolism. Hepatology 1995;21:41-45.

5. Amigo L, Husche C, Zanlungo S, et al. Cholecystectomy increases hepatic triglyceride content and very-low-density lipoproteins production in mice. Liver Int 2011;31:52-64.

6. Ruhl CE, Everhart JE. Relationship of non-alcoholic fatty liver disease with cholecystectomy in the US population. Am J Gastroenterol 2013;108:952-958.

7. Sonne DP, Hare KJ, Martens $\mathrm{P}$, et al. Postprandial gut hormone responses and glucose metabolism in cholecystectomized patients. Am J Physiol Gastrointest Liver Physiol 2013;304:G413-G419.

8. Lefebvre P, Cariou B, Lien F, et al. Role of bile acids and bile acid receptors in metabolic regulation. Physiol Rev 2009;89:147-191.

9. Trauner $M$, Claudel $T$, Fickert $P$, et al. Bile acids as regulators of hepatic lipid and glucose metabolism. Dig Dis 2010;28:220-224.

10. Pols TW, Noriega LG, Nomura $M$, et al. The bile acid membrane receptor TGR5 as an emerging target in metabolism and inflammation. J Hepatol 2011;54:1263-1272.

11. Alberti KG, Eckel RH, Grundy SM, et al. Harmonizing the metabolic syndrome: a joint interim statement of the International Diabetes Federation Task Force on Epidemiology and Prevention; National Heart, Lung, and Blood Institute; American Heart Association; World Heart Federation; International Atherosclerosis Society; and International Association for the Study of Obesity. Circulation 2009;120:1640-1645.

12. Beltrán-Sánchez $\mathrm{H}$, Harhay $M O$, Harhay $M M$, et al. Prevalence and trends of metabolic syndrome in the adult U.S. population, 1999-2010. J Am Coll Cardiol 2013;62:697-703.

13. Ravikiran $M$, Bhansali A, Ravikumar P, et al. Prevalence and risk factors of metabolic syndrome among Asian Indians: a community survey. Diabetes Res Clin Pract 2010;89:181-188.

14. Zuo H, Shi Z, Hu X, et al. Prevalence of metabolic syndrome and factors associated with its components in Chinese adults. Metabolism 2009;58: 1102-1108.

15. Johnson WD, Kroon JJ, Greenway FL, et al. Prevalence of risk factors for metabolic syndrome in adolescents: National Health and Nutrition Examination Survey (NHANES), 2001-2006. Arch Pediatr Adolesc Med 2009;163:371-377.

16. Sattar N, McConnachie A, Shaper AG, et al. Can metabolic syndrome usefully predict cardiovascular disease and diabetes? Outcome data from two prospective studies. Lancet 2008;371:1927-1935.

17. Agrawal V, Shah A, Rice C, et al. Impact of treating the metabolic syndrome on chronic kidney disease. Nat Rev Nephrol 2009;5:520-528.

18. Hamaguchi M, Kojima T, Takeda N, et al. The metabolic syndrome as a predictor of nonalcoholic fatty liver disease. Ann Intern Med 2005;143: 722-728.

19. Alberti KG, Zimmet $P$, Shaw J. The metabolic syndrome-a new worldwide definition. Lancet 2005;366:1059-1062.

20. Grundy SM, Cleeman Jl, Daniels SR, et al. Diagnosis and management of the metabolic syndrome: an American Heart Association/National Heart, Lung, andBlood Institute Scientific Statement. Circulation 2005; 112:2735-2752.

21. Després JP, Lemieux I. Abdominal obesity and metabolic syndrome. Nature 2006:444:881-887.

22. Petersen KF, Dufour S, Savage DB, et al. The role of skeletal muscle insulin resistance in the pathogenesis of the metabolic syndrome. Proc Natl Acad Sci USA 2007;104:12587-12594.

23. Méndez-Sánchez N, Chavez-Tapia NC, Motola-Kuba D, et al. Metabolic syndrome as a risk factor for gallstone disease. World J Gastroenterol 2005;11:1653-1657.

24. Chen $\mathrm{LY}$, Qiao QH, Zhang SC, et al. Metabolic syndrome and gallstone disease. World J Gastroenterol 2012;18:4215-4220.

25. Chang $Y$, Sung $E$, Ryu $S$, et al. Insulin resistance is associated with gallstones even in non-obese, non-diabetic Korean men. J Korean Med Sci 2008;23:644-650.

26. Ata N, Kucukazman M, Yavuz B, et al. The metabolic syndrome is associated with complicated gallstone disease. Can J Gastroenterol 2011;25:274-276.

27. Shen $\mathrm{C}, \mathrm{Wu} \mathrm{X}, \mathrm{Xu} \mathrm{C}$, et al. Association of cholecystectomy with metabolic syndrome in a Chinese population. PLOS ONE 2014;9: e88189.

28. Houghton PW, Donaldson LA, Jenkinson LR, et al. Weight gain after cholecystectomy. Br Med J 1984;289:1350.
29. Nervi F, Miquel JF, Alvarez M, et al. Gallbladder disease is associated with insulin resistance in a high risk Hispanic population. J Hepatol 2006:45:299-305.

30. Ali RB, Cahill RA, Watson RG. Weight gain after laparoscopic cholecystectomy. Ir J Med Sci 2004;173:9-12.

31. Yazdankhah Kenary A, Yaghoobi Notash Jr A, Nazari M, et al. Measuring the rate of weight gain and the influential role of diet in patients undergoing elective laparoscopic cholecystectomy: a 6-month followup study. Int J Food Sci Nutr 2012;63:645-648.

32. Juvonen $\mathrm{T}$, Kervinen $\mathrm{K}$, Kairaluoma MI, et al. Effect of cholecystectomy on plasma lipid and lipoprotein levels. Hepatogastroenterology 1995;42:377-382.

33. Chavez-Tapia NC, Kinney-Novelo IM, Sifuentes-Rentería SE, et al. Association between cholecystectomy for gallstone disease and risk factors for cardiovascular disease. Ann Hepatol 2012;11:85-89.

34. Yun S, Choi D, Lee KG, et al. Cholecystectomy causes ultrasound evidence of increased hepatic steatosis. World J Surg 2016;40: 1412-1421.

35. Ioannou GN. Cholelithiasis, cholecystectomy, and liver disease. Am J Gastroenterol 2010;105:1364-1373.

36. Kwak MS, Kim D, Chung GE, et al. Cholecystectomy is independently associated with nonalcoholic fatty liver disease in an Asian population. World J Gastroenterol 2015;21:6287-6295.

37. Chen Y, Kong J, Wu S. Cholesterol gallstone disease: focusing on the role of gallbladder. Lab Invest 2015;95:124-131.

38. Zhu J, Han TQ, Chen S, et al. Gallbladder motor function, plasma cholecystokinin and cholecystokinin receptor of gallbladder in cholesterol stone patients. World J Gastroenterol 2005;11:1685-1689.

39. Housset $C$, Chrétien $Y$, Debray $D$, et al. Functions of the gallbladder. Compr Physiol 2016;6:1549-1577.

40. Hylemon PB, Zhou H, Pandak WM, et al. Bile acids as regulatory molecules. J Lipid Res 2009;50:1509-1520.

41. de Aguiar Vallim TQ, Tarling EJ, Edwards PA. Pleiotropic roles of bile acids in metabolism. Cell Metab 2013;17:657-669.

42. Kuipers F, Bloks VW, Groen AK. Beyond intestinal soap-bile acids in metabolic control. Nat Rev Endocrinol 2014;10:488-498.

43. Matsubara T, Li F, Gonzalez FJ. FXR signaling in the enterohepatic system. Mol Cell Endocrinol 2013;368:17-29.

44. Zhang Y, Lee FY, Barrera G, et al. Activation of the nuclear receptor FXR improves hyperglycemia and hyperlipidemia in diabetic mice. Proc Natl Acad Sci USA 2006;103:1006-1011.

45. Cariou B, van Harmelen K, Duran-Sandoval D, et al. The farnesoid X receptor modulates adiposity and peripheral insulin sensitivity in mice. J Biol Chem 2006;281:11039-11049.

46. Ma K, Saha PK, Chan L, et al. Farnesoid X receptor is essential for normal glucose homeostasis. J Clin Invest 2006;116:1102-1109.

47. Pols TW, Noriega LG, Nomura $M$, et al. The bile acid membrane receptor TGR5: a valuable metabolic target. Dig Dis 2011;29:37-44.

48. Bunnett NW. Neuro-humoral signalling by bile acids and the TGR5 receptor in the gastrointestinal tract. J Physiol 2014;592: 2943-2950.

49. Poole DP, Godfrey C, Cattaruzza F, et al. Expression and function of the bile acid receptor GPBAR1 (TGR5) in the murine enteric nervous system. Neurogastroenterol Motil 2010;22(814-25): e227-e228.

50. Maruyama T, Tanaka K, Suzuki J, et al. Targeted disruption of G proteincoupled bile acid receptor 1 (Gpbar1/M-Bar) in mice. J Endocrinol 2006;191:197-205.

51. Shaham O, Wei R, Wang TJ, et al. Metabolic profiling of the human response to a glucose challenge reveals distinct axes of insulin sensitivity. Mol Syst Biol 2008:4:214.

52. Patti ME, Houten SM, Bianco AC, et al. Serum bile acids are higher in humans with prior gastric bypass: potential contribution to improved glucose and lipid metabolism. Obesity 2009;17:1671-1677.

53. Thomas C, Auwerx J, Schoonjans K. Bile acids and the membrane bile acid receptor TGR5-connecting nutrition and metabolism. Thyroid 2008;18:167-174.

54. Keitel V, Häussinger D. Perspective: TGR5 (Gpbar-1) in liver physiology and disease. Clin Res Hepatol Gastroenterol 2012;36:412-419.

55. Thomas C, Gioiello A, Noriega L, et al. TGR5-mediated bile acid sensing controls glucose homeostasis. Cell Metab 2009;10:167-177.

56. Oldham-Ott CK, Gilloteaux J. Comparative morphology of the gallbladder and biliary tract in vertebrates: variation in structure, 
homology in function and gallstones. Microsc Res Tech 1997;38: 571-597.

57. Zweers SJ, Booij KA, Komuta $M$, et al. The human gallbladder secretes fibroblast growth factor 19 into bile: towards defining the role of fibroblast growth factor 19 in the enterobiliary tract. Hepatology 2012;55:575-583.

58. Tomlinson E, Fu L, John L, et al. Transgenic mice expressing human fibroblast growth factor-19 display increased metabolic rate and decreased adiposity. Endocrinology 2002;143:1741-1747.

59. Fu L, John LM, Adams SH, et al. Fibroblast growth factor 19 increases metabolic rate and reverses dietary and leptin-deficient diabetes. Endocrinology 2004;145:2594-2603.
60. Jones S. Mini-review: endocrine actions of fibroblast growth factor 19 . Mol Pharm 2008;5:42-48.

61. Bhatnagar S, Damron HA, Hillgartner FB. Fibroblast growth factor-19, a novel factor that inhibits hepatic fatty acid synthesis. J Biol Chem 2009;284:10023-10033.

62. Barrera F, Azócar L, Molina H, et al. Effect of cholecystectomy on bile acid synthesis and circulating levels of fibroblast growth factor 19. Ann Hepatol 2015;14:710-721.

63. Keitel V, Cupisti K, Ullmer C, et al. The membrane-bound bile acid receptor TGR5 is localized in the epithelium of human gallbladders. Hepatology 2009;50:861-870. 